NASA Technical Memorandum 102126

\title{
Some Characteristics of Bypass Transition in a Heated Boundary Layer
}

\author{
K.H. Sohn \\ Case Western Reserve University \\ Cleveland, Ohio \\ J.E. O'Brien \\ Lewis Research Center \\ Cleveland, Ohio \\ and \\ E. Reshotko \\ Case Western Reserve University \\ Cleveland, Ohio
}

Prepared for the

Seventh Symposium on Turbulent Shear Flows sponsored by Turbulent Shear Flow Committee Stanford, California, August 21-23, 1989

\section{N/SA}

(NASA-TH-102126) SOME CHARACTERISTICS OF BYPASS TRANSITION IN A HEATED BOUNDARY LAYER

(NASA. Lewis Research Center) 8 p CSCL 20D 


\title{
SOME CHARACTERISTICS OF BYPASS TRANSTTION IN A HEATED BOUNDARY LAYER
}

\author{
K. H. Sohn', J. E. O'Brien ${ }^{2}$, E. Reshotko' \\ ${ }^{1}$ Department of Mechanical and Aerospace Engineering \\ Case Western Reserve University \\ 2 NASA Lewis Research Center
}

\section{ABSTRACT}

Experimental measurements of both mean and conditionally sampled characteristics of laminar, transitional and low Reynolds number turbulent boundary layers on a heated flat plate are presented. Measurements were obtained in air over a range of freestream turbulence intensities from $0.3 \%$ to $6 \%$ with a freestream velocity of $30.5 \mathrm{~m} / \mathrm{s}$ and zero pressure gradient. Conditional sampling performed in the transitional boundary layers indicate the existence of a near-wall drop in intermittency, especially pronounced at low intermittencies. Non-turbulent intervals were observed to possess large levels of low-frequency unsteadiness, and turbulent intervals had peak intensities as much as $50 \%$ higher than were measured at fully turbulent stations. Heat transfer results were consistent with results of previous researchers and Reynolds analogy factors were found to be well predicted by laminar and turbulent correlations which accounted for unheated starting length. A small dependence of the turbulent Reynolds analogy factors on freestream turbulence level was observed. Laminar boundary layer spectra indicated selective amplification of unstable frequencies. These instabilities appear to play a dominant role in the transition process only for the lowest freestream turbulence level studied, however.

\section{INTRODUCTION}

Boundary layer transition in a low-disturbance environment is characterized by a well-documented progression of initial amplification of two-dimensional Tollmien-Schlichting waves followed by the formation of three-dimensional structures and subsequent development of turbulent spots. Linear stability theory has been used to predict the critical Reynolds number above which amplification of small (linear) disturbances may occur, leading to the progression described above. Morkovin (1978) introduced the term bypass transition to describe the transition process which occurs in the presence of larger (nonlinear) disturbances. In the bypass transition process, the formation of turbulent spots may occur without the initial amplification of linear waves. Bypass transition occurs in high-disturbance flows such as those present in gas turbines.

Previous studies of the influence of freestream turbulence on boundary layer transition were primarily aimed at measuring mean characteristics of transitioning boundary layers (eg., Blair, 1982; Wang et al., 1985). The present study, along with several other very recent studies (Blair, 1988; Kuan and Wang, 1988; Kim et al., 1989), focuses additionally on determination of the separate statistics of the respective turbulent and non-turbulent parts of these intermittent boundary layers using conditional sampling techniques. Limited results of conditional sampling in a transitional boundary layer were first reported by Arnal et al. (1978). Detailed studies of individual turbulent spot structure have also been performed using artificially generated turbulent spots in otherwise laminar boundary layers (Antonia et al., 1981; Cantwell et al., 1978; Wygnanski et al., 1976). Results of these studies, while elegant, are only applicable in the very early stages of transition, before coalescence of multiple spots occurs. The turbulent spots of the present study arose and merged "naturally" as a result of freestream turbulence.

The purpose of this study was to measure some of the characteristics of the boundary layer transition process on a heated flat plate for conditions which span a range of freestream turbulence levels. The highest freestream turbulence levels studied were unambiguous bypass cases since transition occurred at Reynolds numbers lower than the minimum critical Reynolds number predicted from linear stability theory. Detailed probing of the pre-transitional boundary layers was not possible in these cases, however, since transition occurred very close to the leading edge of the test surface. Intermediate turbulence levels produced post-critical transitions. These cases may still be termed bypass, however in the sense that they do not follow the classical progression described above for low-disturbance environments. Detailed measurements presented in the present study will focus on these cases. The lowest freestream turbulence case studied produced a transition process which was dominated by the formation and subsequent breakdown of TS-type waves (see Suder et al., 1988).

\section{THE EXPERIMENTS}

The experimental program was carried out in a closed-loop wind tunnel. This tunnel is similar in design to the tunnel of Blair et al. (1981). The tunnel test section is rectangular in cross section and measures $15.2 \mathrm{~cm} \mathrm{high,68.6}$ $\mathrm{cm}$ wide and $152.4 \mathrm{~cm}$ long. The smooth floor of the test section, which was uniformly heated using an inconel foil heater $(0.025 \mathrm{~mm}$ foil thickness), serves as the measurement test surface. Surface temperatures were monitored by means of thermocouples spot-welded to the back side of the foil, which was bonded to a $5.08 \mathrm{~cm}$ thickness of polyurethane foam. At the entrance to the test section, a series of two upstream-facing scoops were used to bleed off the boundary layer which develops in the contraction nozzle. This arrangement results in a short unheated starting length (3.5 $\mathrm{cm})$. The boundary layer which grows on the test surface is not intentionally excited in any way, other than by the freestream turbulence, and transition occurs naturally, with random arrival of turbulent spots at a given measurement station. Upstream of the test section and nozzle, a flow-conditioning section serves to remove any large-scale nonuniformities introduced by the blower and to reduce the freestream turbulence level. At the downstream end of the flow-conditioning section and upstream of the nozzle contraction, turbulence grids (rectangular-bar, $62 \%$ open area) could be positioned in order to set the freestream turbulence level in the test section. Six freestream turbulence conditions were available, ranging from $0.3 \%$ to $6 \%$. The roof of the test section was adjustable in order to allow establishment of a desired streamwise pressure gradient. The experiments described herein were carried out with a freestream velocity of $30.5 \mathrm{~m} / \mathrm{s}$ and zero pressure gradient. Further details on the 
facility may be obtained from Suder et al. (1988).

Boundary layer measurements of mean and fluctuating velocity and temperature were obtained along the tunnel centerline. Additional profile and intermittency measurements obtained at selected off-centerline locations verified tunnel spanwise uniformity. Surface measurements of mean heat flux and temperature were also acquired. Both overall and conditionally sampled measurements were made. Velocity and temperature profiles were acquired using a commercially available boundary-layer hot wire probe (TSI model 1218). For the present study, boundary layer velocity and temperature profiles were obtained at streamwise locations in the range $12.7 \mathrm{~cm}<\mathrm{x}<50.8 \mathrm{~cm}$. Typical boundary layer thicknesses were of the order of $6 \mathrm{~mm}$ and the minimum y value for each profile was about $0.15 \mathrm{~mm}$.

Steady-state tunnel operating conditions were monitored by means of a multichannel data acquisition system which provided a control-room display of steady-state quantities such as temperatures, pressures, freestream velocity, etc. in engineering units with updates approximately every two seconds. Time-averaged velocity data (mean and rms) were acquired using a separate precision averaging digital multimeter set for 200 averages for each data point. High-speed data acquisition was accomplished using a multichannel 12-bit digital waveform recorder. The sampling rate was set at $50 \mathrm{kHz}$ and $32 \mathrm{kbytes}$ of data were stored for each waveform. Spectral data were acquired using a 1024-point 12-bit digital FFT analyzer with a sampling rate of $5 \mathrm{kHz}$. Each spectral curve represents at least 100 averages.

\section{RESULTS}

Detailed heat transfer and boundary layer measurements have been obtained for six different levels of freestream turbulence. Nominal values of freestream turbulence intensity and integral length scale, $\Lambda$, for the various grid configurations are listed in Table 1. Detailed profiles presented in the present paper will focus on results obtained using grid 1 . This case was chosen as the focus because the transition zone for this turbulence level overlaps the region in which detailed measurements were obtained. Further details of the freestream turbulence measurements are available in Suder et al. (1988).

Momentum Boundary Layer. Typical development of the momentum boundary layer is illustrated in Figure 1 . In this figure, solid curves representing $\mathrm{u}^{+}=\mathrm{y}^{+}$, the Blasius solution corresponding to three of the upstream measurement stations, and the Musker (1979) continuous law of the wall for a fully turbulent boundary layer are shown along with the experimental data. These profiles span nearly the entire range from laminar to fully turbulent flow. Note the excellent agreement of the two farthest upstream profiles with the Blasius curves. Values for $u$ required to construct this plot were inferred from the near-wall data points $\left(\mathrm{y}^{+}<10\right)$ where, in all cases, the data are tightly bounded by $u^{+}=y^{+}$and the Musker (1979) continuous law of the wall. In addition, the laminar values were checked against laminar theory and the fully turbulent cases were analyzed using a Clauser fit technique. A plot of the experimentally determined skin friction coefficients, $C_{f}$, versus momentum thickness Reynolds

\begin{tabular}{c|c|c} 
Grid $\#$ & $u^{\prime} / \mathrm{U}_{e}(8)$ & $\Lambda(\mathrm{cm})$ \\
\hline .5 & 0.6 & 0.8 \\
1 & 1.0 & 1.3 \\
2 & 2.0 & 1.5 \\
3 & 4.2 & 2.1 \\
4 & 5.0 & 2.3
\end{tabular}

Table 1. Nominal grid turbulence characteristics. number for the entire set of freestream turbulence values is shown in Figure 2. Also shown in the figure are solid lines corresponding to laminar theory and a fully turbulent correlation (Schlichting, 1979). Transition is observed to occur at increasingly lower values of Reynolds number as the freestream turbulence level is increased.

Conditionally sampled boundary layer profiles were also acquired. The procedure used for accomplishing the necessary laminar/turbulent decisions is illustrated in Figure 3. The top trace in the figure is a small portion of a digitally recorded near-wall velocity record (grid $1, y \approx 0.15 \mathrm{~mm}$ ). The velocity trace reveals some of the interesting characteristics of an intermittent boundary layer. For example, upon passage of a turbulent spot, the velocity achieves a level characteristic of a turbulent boundary layer very quickly, whereas following the return to laminar transport, there is a relatively long time period required for relaxation to the "steady" laminar level. This behavior is indicative of the much higher transport efficiency associated with turbulence. It also indicates a potential stumbling block for simple intermittency-weighted transition models which assume Blasius-type behavior during laminar periods and fully-developed turbulent behavior during turbulent periods. The second trace in Figure 3 is the square of the second derivative of the velocity trace. Nine-point smoothing is then applied, resulting in the third trace after which an appropriate threshold value is selected which results in the bottom trace, the indicator function. The square of the first derivative is also treated similarly in order to eliminate false laminar indications caused by second derivative zero-crossings. Attempts were made to adopt a completely numerical method to quantify the threshold selection process using the cumulative distribution function approach described

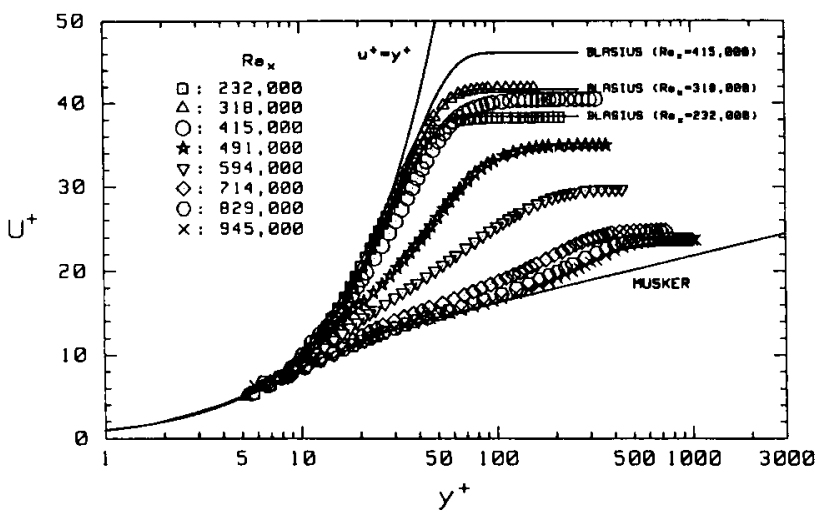

Figure 1. Mean velocity profiles, grid 1.

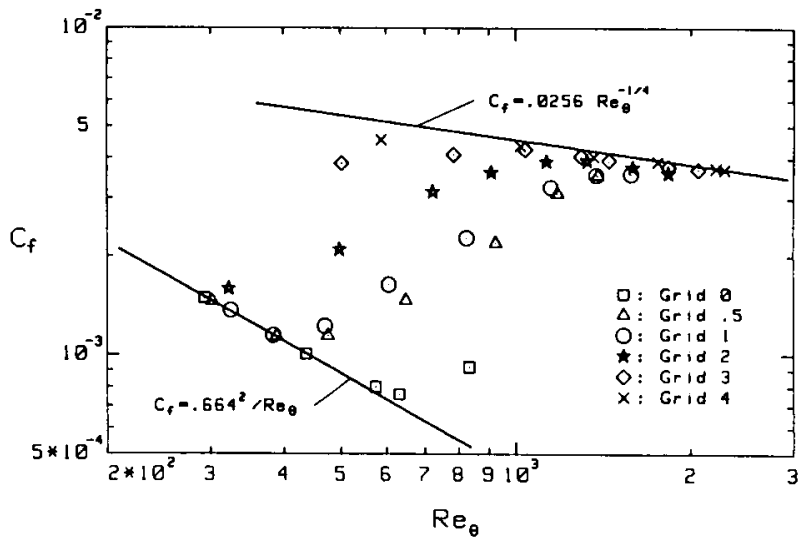

Figure 2. Skin friction coefficients. 


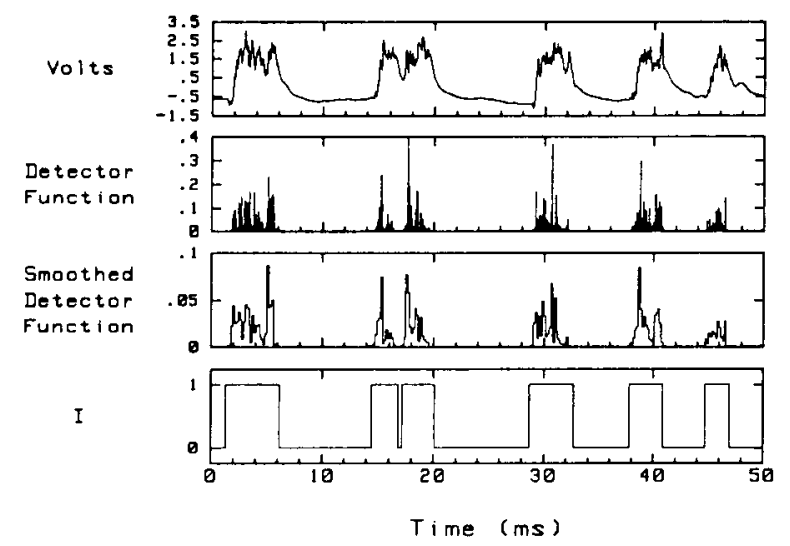

Figure 3. Technique for determination of indicator function.

by Hedley and Keffer (1974). This method proved only partially successful and fine tuning of the threshold values was required at each individual measurement location ( $x$ and $y$ ) in order to obtain an accurate indicator function as determined by careful direct comparison of each indicator function with the actual intermittent waveforms.

Determination of the indicator function allows for calculation of an intermittency value, $\Gamma$, (and its variation through the boundary layer) and for discrimination between turbulent and non-turbulent portions of the velocity record. Boundary layer intermittency profiles obtained with grids 1 and 2 are shown in Figure $4(a, b)$ along with the error-function distribution of Klebanoff (1955) for a fully developed turbulent boundary layer. Consistent with recent results obtained by Kuan and Wang (1988), these profiles do not decrease monotonically with $y$. Instead, a peak is observed at $y / \delta^{*} \approx 1$, with lower intermittencies very near the wall. For the farther downstream, higher intermittency cases, the peak is followed by a plateau of nearly constant intermittency. This near-wall drop-off in intermittency is consistent with turbulent spot elevation cross-sectional shapes which exhibit leading and trailing-edge overhangs as observed by Cantwell et al (1978) and others. The data of Blair (1988), acquired in a moderately accelerating boundary layer, do not exhibit a well-defined near-wall intermittency drop-off, however.

Conditionally sampled mean velocity profiles obtained in the intermittent boundary layer for grid 1 are shown in Figure $5(a-c)$. In each of the figures, three profiles are shown. The non-turbulent profiles represent the mean of the velocities obtained during times when the indicator function was zero. It should be noted that these times included the post-burst relaxation period during which the transport is laminar, but the magnitude of the instantaneous velocities may be more

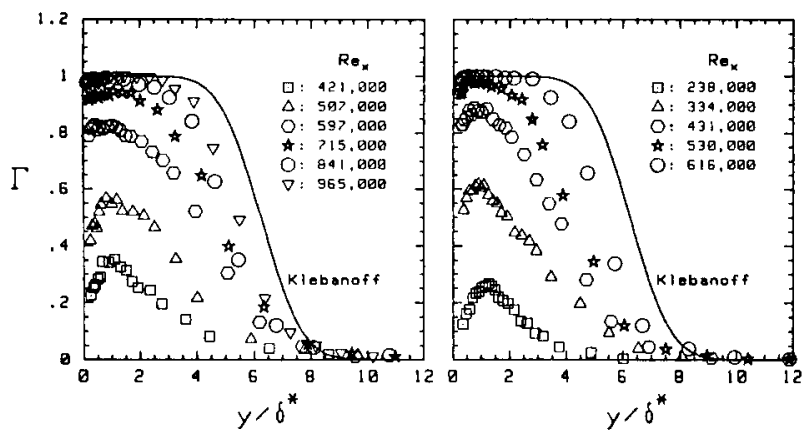

Figure 4. Intermittency profiles; (a) grid 1; (b) grid 2. characteristic of the turbulent part of the flow (see Fig. 3). For comparison, the corresponding Blasius curves are also shown. The middle profile shown in each case represents the overall velocity profile as determined from a direct time average of the digitally recorded data. The turbulent profiles were obtained from an average of the velocity data acquired during times when the indicator function was equal to one.

Low-intermittency non-turbulent profiles agree well with corresponding Blasius profiles. As the intermittency increases, however, the non-turbulent profiles of Figure 5 increasingly deviate from the Blasius curves, falling well below the Blasius values for the highest intermittency case (Figure 5c). The turbulent profiles have the appearance of low Reynolds number turbulent boundary layers with a large wake region, especially at low intermittencies.

Boundary layer profiles of the overall apparent streamwise component of turbulence intensity for grid 1 are presented in Figure 6. Recall that the mean profiles for this case exhibited essentially the entire range of behavior from laminar to fully turbulent. The farthest upstream profiles are typical for laminar boundary layers in the presence of freestream turbulence with a peak unsteadiness value occurring at

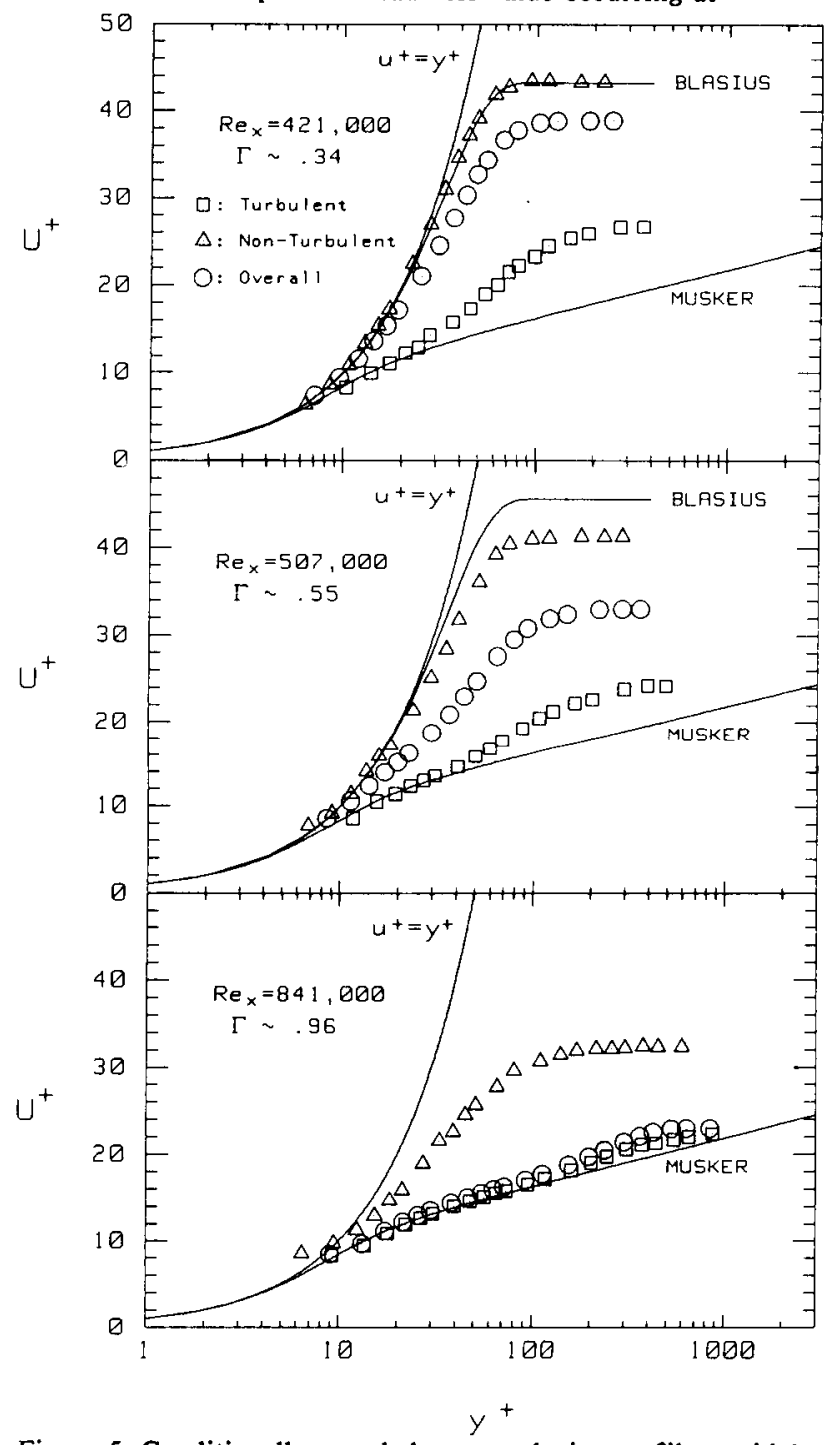

Figure 5. Conditionally sampled mean velocity profiles, grid 1. 
$y / \delta^{*}=1.3$. A double peak in the rms profiles occurs at measurement stations located in the transition region. It is especially pronounced in the later stages of transition $(\Gamma \approx 0.8)$ and lingers even into the immature stages of the turbulent boundary layer. This double peak will be discussed further in conjunction with presentation of the conditionally sampled turbulence intensity profiles. In addition, the magnitude of the near-wall peak is much larger in the transition region than farther downstream. This behavior has been shown to be typical for transitioning boundary layers (Arnal et al., 1978; Suder et al., 1988; Kuan and Wang, 1988; Blair, 1988; Kim et al., 1989).

Conditionally sampled streamwise turbulence intensity (rms) profiles are shown in Figure $7(a-c)$ for the same conditions as for Figure 5. These rms values were calculated from the digitally recorded velocity records relative to the respective mean values for the turbulent and non-turbulent parts of the flow, thereby eliminating the mean-step rms contribution associated with switching between the turbulent and non-turbulent levels. Reminiscent of the laminar overall rms profiles presented in Figure 6 for the farthest upstream stations, the non-turbulent conditionally sampled rms profiles of Figure 7 have a rounded shape and exhibit a peak at $y / \delta^{*} \simeq 1.3$. The peak magnitudes are greater, however, than were observed for any of the strictly laminar profiles and increase significantly with downstream distance, exceeding both the turbulent and the overall profiles at $y / \delta^{*} \approx 1$ and $\operatorname{Re}_{\mathrm{x}} \geq 597,000$. This observation was supported by direct inspection of the intermittent waveforms which revealed high levels of low-frequency unsteadiness in the non-turbulent intervals between turbulent spots, especially pronounced near $y / \delta^{*}=1$. The turbulent part of the rms profiles also have a shape which is similar to the shape expected for a fully turbulent boundary layer, with a peak very near the wall $\left(y / \delta^{*} \approx 0.5\right)$ followed by a relatively flat region and a final drop of $f$ to the freestream value outside of the boundary layer. The magnitude of the near-wall turbulence intensity, however, is highest for the lowest intermittency case, and decreases significantly with downstream distance. This result indicates that transport processes occurring in the turbulent spots will not be well modeled by standard turbulence models used in equilibrium turbulent boundary layers.

Values of overall apparent turbulence intensity in excess of a direct intermittency-weighted average of the respective turbulent and non-turbulent parts are observed both very near the wall $\left(y / \delta^{*} \simeq 0.6\right)$ and also farther out in the boundary layer $\left(2<y / \delta^{*}<4\right)$, indicating the presence of a mean-step contribution to the overall rms value. This mean-step contribution is associated with the significantly different mean values of the turbulent and non-turbulent velocities at these $y$

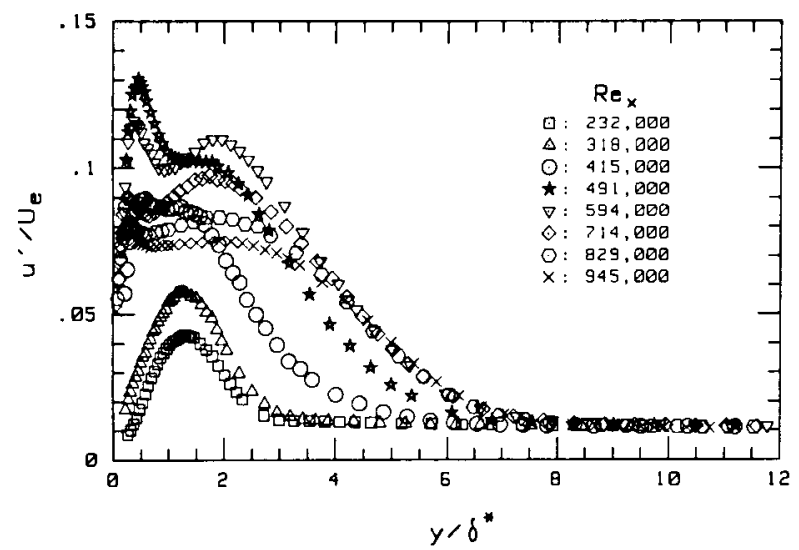

Figure 6. Overall streamwise rms velocity profiles, grid 1. locations. In fact, the overall rms value actually exceeds the turbulent rms value for several near-wall stations (Figure 7 b). At the near-wall stations, the velocity records are characterized by large positive excursions during the passage of a turbulent spot and correspondingly large apparent overall rms values are observed. Similarly, at y measurement stations located farther out in the boundary layer, large negative velocity excursions occur during the passage of a turbulent spot, resulting in a large mean-step contribution to the second peak in the overall rms profiles observed at these y locations.

Thermal Boundary Layer. A representative series of boundary layer temperature profiles corresponding to the velocity profiles of Figure 1 is shown in Figure 8. A solid line representing the theoretical laminar temperature profile corresponding to the farthest upstream station, including a correction for unheated starting length is also shown. These data exhibit behavior which is qualitatively similar to the velocity profiles. A turbulent Prandtl number of 0.9 and a conduction layer thickness of 13.2 were assumed in obtaining the fully turbulent line shown in the figure. A slightly higher value of $\operatorname{Pr}_{t}$ may be required for a best fit to the early

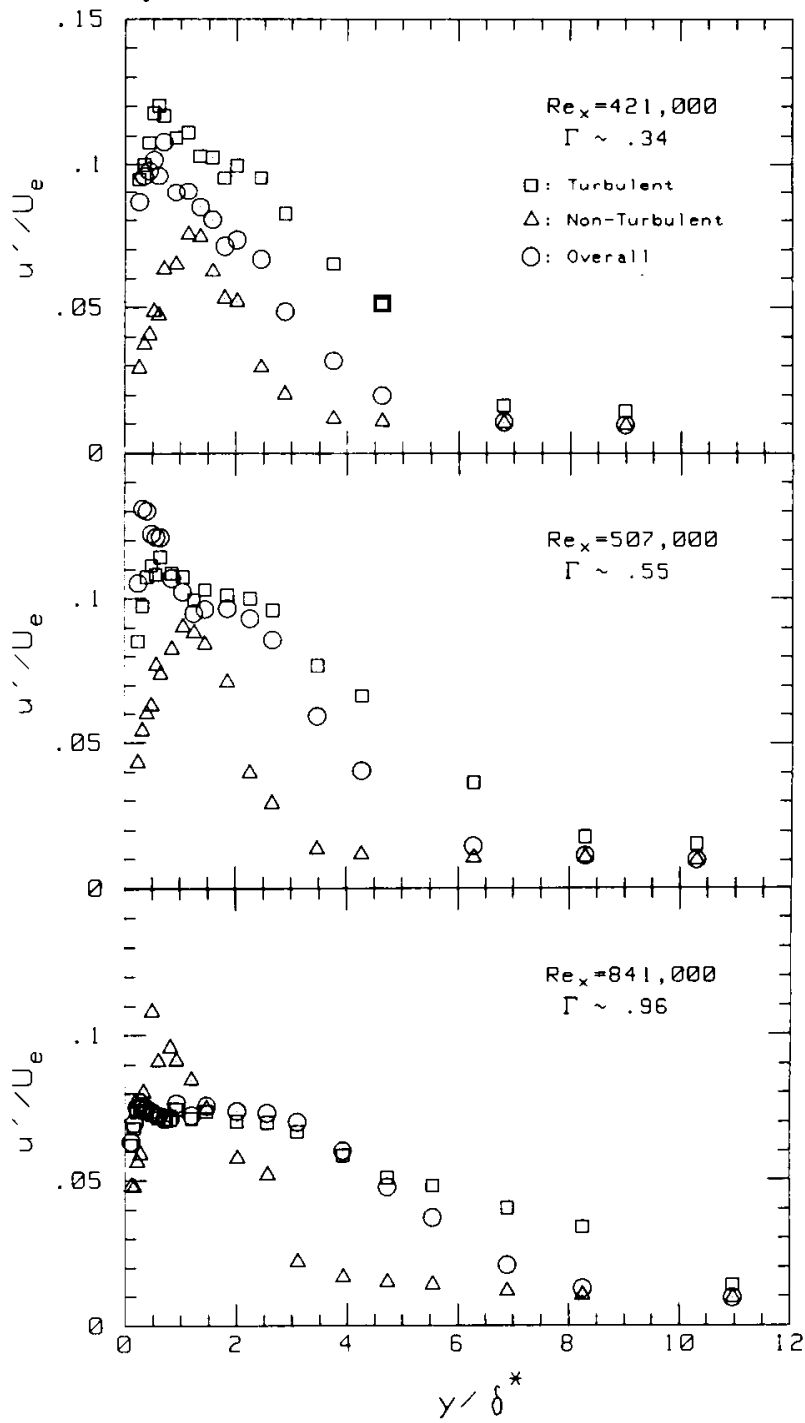

Figure 7. Conditionally sampled rms velocity profiles, grid 1. 


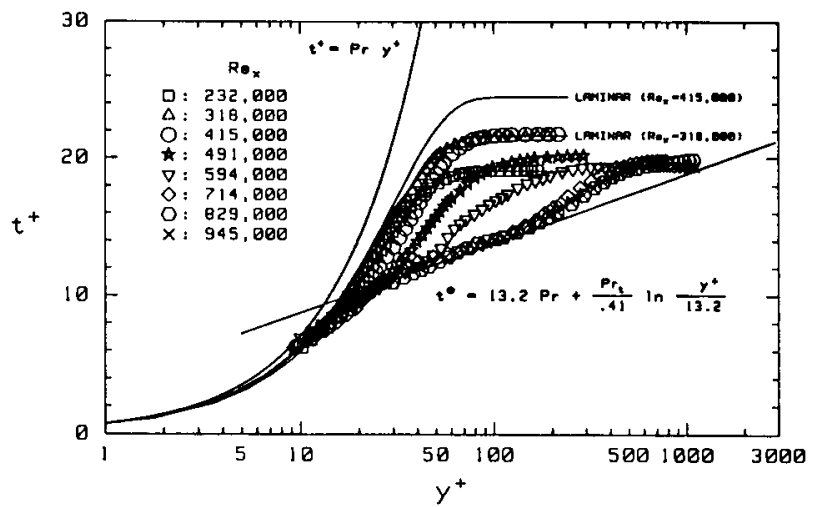

Figure 8. Mean temperature profiles, grid 1.

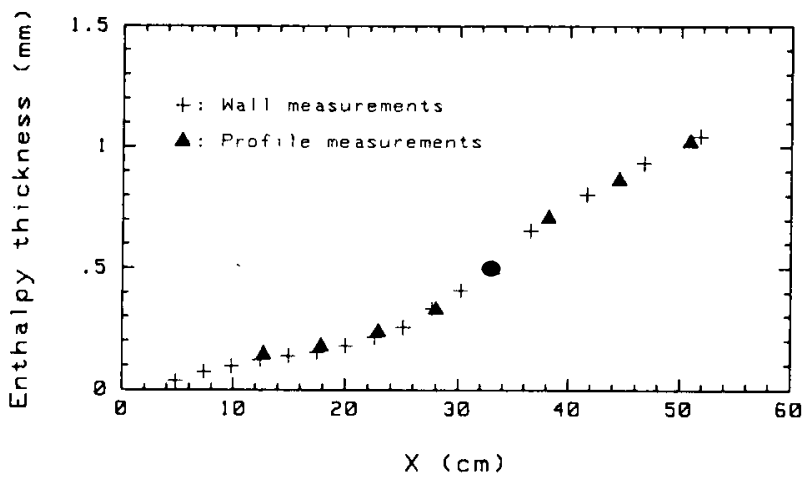

Figure 9. Enthalpy thickness and energy closure, grid 1 .

post-transition turbulent profiles (see Wang et al., 1985). In order to check for energy closure, enthalpy thickness values were calculated from the boundary layer temperature profiles and compared to values obtained from direct integration of the wall heat flux. This comparison is shown in Figure 9. The agreement is excellent, indicating consistency between the wall measurements and the profile measurements. Thermal boundary layer development is also depicted.

Stanton number distributions, which were obtained directly from wall heat flux and temperature measurements, are presented in Figure 10. A correction for radiation heat loss was also included. These data agree very well with the data of Blair (1983), both in terms of magnitude and transition location. The lower solid line is the theoretical laminar Stanton number including a correction for unheated starting length. The upper solid line is the turbulent correlation given by Kays and Crawford (1980).

A quantity which combines information from both the momentum and thermal boundary layers is the Reynolds analogy factor, $2 S t / C_{f}$. A plot of experimentally determined Reynolds analogy factors are presented in Figure 11 . The solid curves shown on the figure represent expected Reynolds analogy factors for the laminar and turbulent regimes, accounting for thermal boundary conditions and unheated starting length. Appropriate laminar theoretical results and turbulent correlations from Kays and Crawford (1980) were combined in order to obtain the curves shown. In the laminar region, the Stanton number is very sensitive to thermal boundary condition and the uniform wall heat flux of the present experiment results in a Stanton number augmentation of $34 \%$ (Kays and Crawford, 1980) over the uniform wall temperature case. Furthermore, the unheated starting length of the test surface produces an additional augmentation, especially important for the upstream stations. Consequently, Reynolds analogy factors as high as 2.0 were observed in the laminar region. Note the excellent agreement of the laminar data with the laminar prediction. For turbulent boundary layers, heat transfer results are much less sensitive to both the thermal boundary condition and the unheated starting length, and values much closer to the "standard" $\mathrm{Pr}^{-2} / 3$ value ( 1.27 for $\operatorname{Pr}=0.7$ ) are observed. The data for the high-turbulence cases are generally better predicted by $\mathrm{Pr}^{-2 / 3}$, while the turbulent heat transfer data for the lower freestream turbulence cases are closer to the lower Kays and Crawford curve. This slight increase of turbulent Reynolds analogy factor with freestream turbulence is consistent with the results of Blair (1983).

Spectra. One-dimensional boundary layer spectra were also acquired. A series of power spectral density curves for grid 1 is presented in Figure 12. Recall that the farthest upstream measurement stations for grid 1 exhibit Blasius-type mean profiles and no evidence of turbulence. Note also that even the farthest upstream measurement location ( $R e_{\text {. }}$ 232,$000 ; \mathbf{R e}^{*} \approx 840$ ) is post-critical in terms of linear stabifity theory. The approximate range of unstable frequencies for this Reynolds number is indicated on the figure. All of the spectra presented in the figure were obtained with the hot wire at its closest proximity to the test surface $(y \propto 0.13 \mathrm{~mm})$ and no conditional sampling was applied.

Within the unstable frequency range, a broadband hump is observed in the power spectrum. In addition, a narrow peak is observed at $930 \mathrm{~Hz}$. This peak was not related to any intentional excitation and was also present in $\mathrm{grid} 0$ and $\mathrm{grid}$ .5 spectra and is therefore not grid related. It also did not appear in spectra obtained at the maximum rms elevation. For the grid 0 case, selective amplification appeared to be centered near this $930 \mathrm{~Hz}$ frequency, with the peak value shifted to

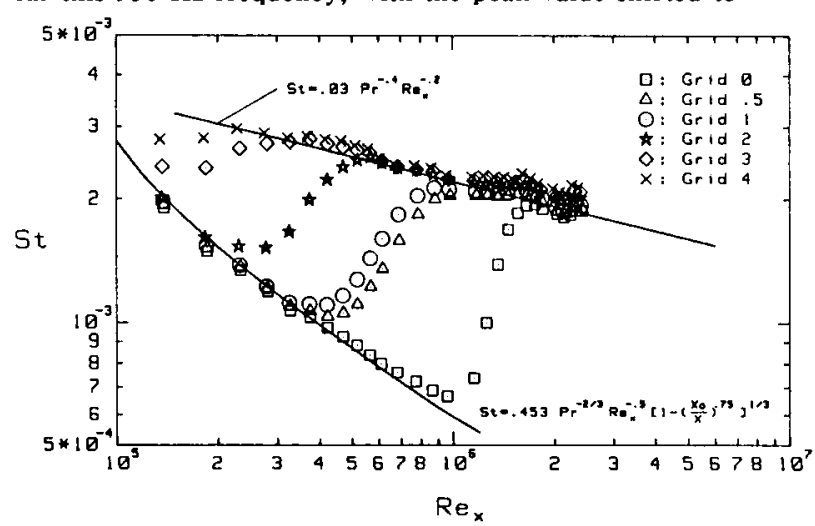

Figure 10. Stanton number distribution.

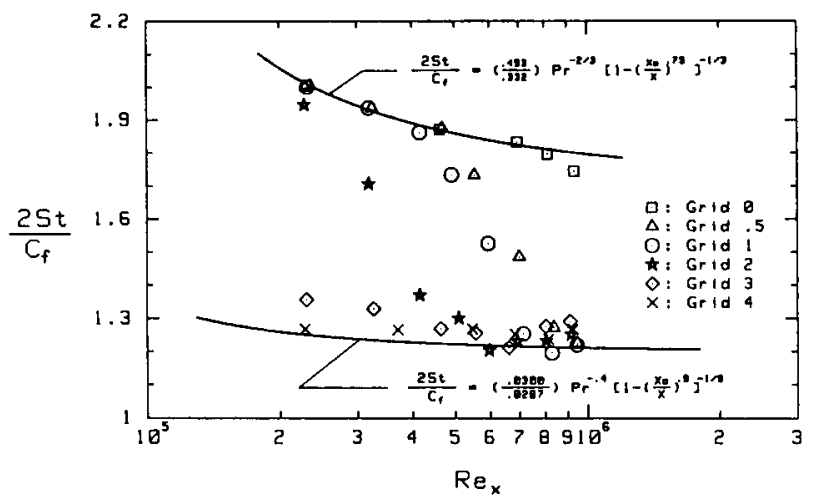

Figure 11. Reynolds analogy factor. 
slightly lower frequencies at farther downstream stations. For the grid 1 case, however, preferred bandwidth amplification is more broadband and is centered in the $1200-1300 \mathrm{~Hz}$ range, again shifting to slightly lower frequencies with increasing downstream distance as expected from linear stability theory. These spectra indicate that receptivity and selective amplification of disturbance frequencies in the range predicted by linear stability theory can occur in laminar boundary layers perturbed by moderate levels (i.e. $1 \%$ ) of freestream turbulence. The waves generated in this process are localized very near the wall, however, and do not seem to play a dominant role in the ultimate transition process at this level of freestream turbulence. With low disturbance levels (eg., grid 0 ), however, the selective amplification of TS-type waves was clearly shown to be the dominant mechanism in the process leading to the formation of turbulent spots (Suder et al., 1988).

Spectra obtained farther out in the laminar boundary layers at elevations corresponding to the maximum observed rms values do not show any clear evidence of selective amplification. This result is consistent with the observations of Blair (1988). Apparently the large-amplitude, lowfrequency unsteadiness present at the maximum rms elevation in the perturbed laminar boundary layers is not related to the unstable frequencies predicted by linear stability theory.

At streamwise measurement locations where intermittency is first observed (eg., curve 4 in Figure 12) the power spectrum is characterized by broadband high-frequency content and a distinct low-frequency hump $(<200 \mathrm{~Hz})$ associated with passage of turbulent spots. Further downstream, as the intermittency approaches 1.0 , this low-frequency hump disappears.

\section{SUMMARY AND CONCLUSIONS}

As part of an ongoing research program focusing on the boundary layer transition process in the presence of freestream disturbances, detailed measurements of the momentum and thermal boundary layers and surface heat transfer on a heated flat plate have been obtained. Conditional sampling was applied as a means of segregating the turbulent and non-turbulent statistics of the intermittent boundary layers.

Excellent agreement of the measured boundary layer mean velocity profiles with laminar theory and turbulent correlations was observed, along with intermediate transitional profiles. Intermittency profiles obtained in the transitional boundary layers exhibited a peak at $y / \delta^{*} \approx 1$, with lower intermitrencies near the wall. These observations are consistent with turbulent spot shapes observed in previous studies which indicated a maximum spot length away from the wall. Non-turbulent

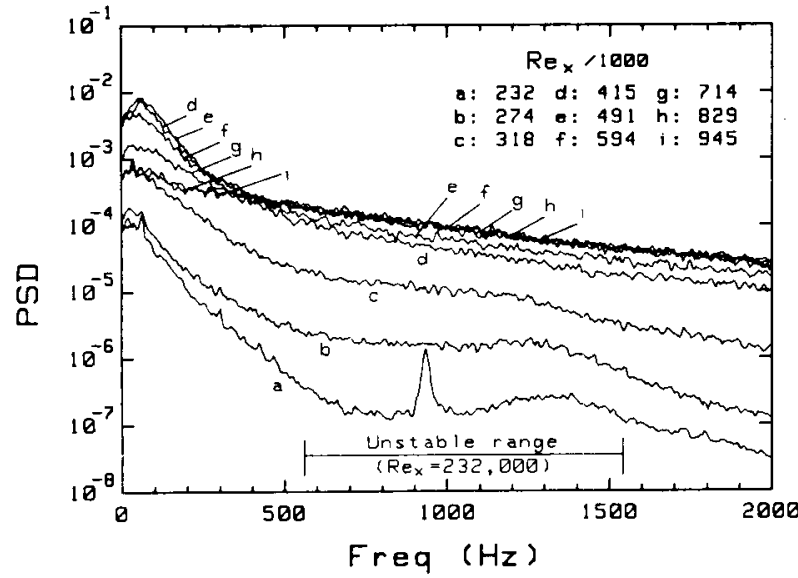

Figure 12. Wall spectra through transition, grid 1. conditionally sampled mean velocity profiles had Blasius-type shapes, but were observed to deviate increasingly from corresponding Blasius profiles with increasing intermittency. Turbulent conditionally sampled mean profiles had the appearance of low-Reynolds number turbulent boundary layers with a large wake region. Conditionally sampled rms velocity profiles revealed high levels of low-frequency unsteadiness in the non-turbulent part of the intermittent boundary layers. The turbulent part of these transitional boundary layers possessed peak turbulence intensities as much as $50 \%$ higher than corresponding fully murbulent values. Thermal boundary layer temperature and surface heat transfer measurements obtained in this study were in excellent agreement with previous studies. Measured Reynolds analogy factors were found to be well predicted by combining appropriate correlations for the respective laminar and turbulent regimes. including corrections for unheated starting length. Boundary layer spectra indicated that some selective amplification of disturbances in the linear instability frequency range predicted was occurring before the onset of turbulent spot formation. For the $1 \%$ freestream turbulence case, however, the waves involved were localized very near the wall and do not seem to play a role in the ultimate breakdown to turbulence.

\section{References}

ANTONIA, R.A. CHAMBERS, A.J., SOKOLOV, M. \& VAN ATTA, C.W. 1981, Simultaneous temperature and velocity measurements in the plane of symmetry of a transitional turbulent spot, J. Fluid Mech. 108,317

ARNAL, D., JUILLEN, J.C. \& MICHEL, R., 1978 Experimental analysis and computation of the onset and development of the boundary layer transition, NASA development

BLAIR, M.F., BAILEY, D.A. \& SCHLINKER, R.H., 1981 , Development of a large-scale wind tunnel for the simulation of turbomachinery airfoil boundary layers, ASME J. Engr. for Power, 103,678 .

BLAIR, M.F., 1982, Influence of free-stream turbulence on boundary layer transition in favorable pressure gradients. ASME J. Engr. for Power, 104, 743 .

BLAIR, M.F., 1983, Influence of free-stream turbulence on turbulent boundary layer heat transfer and mean profile development, Parts I \& II, ASME J. Heat dransfer. 105,33 .

BLAIR, M.F., 1988, Bypass-mode boundary layer transition in accelerating flows, submitted to J. Fluid Mech. November

CANTWELL, B.J., COLLS, D. \& DIMOTAKIS, P.E., 1978 Structure and entrainment in the plane of symmetry of

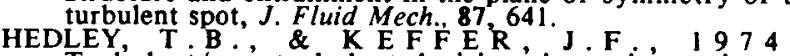
Turbulent/non-turbulent decisions in an intermittent

flow, J. Fluid Mech. 64, part 4, 625.
KAYS, W.M. \& CRAWFORD, M.E. 1980 Convective Heat and Mass Transfer, 2nd ed. McGraw-Hill, New York.

KIM, J., SIMON, T.W. \& KESTORAS, M., 1989 , Flu id mechanics and heat transfer measurements in transitional boundary layers conditionally sampled on intermittency, to be presented at the 1989 ASME National Heat Transfer Conference, Philadelphia.

KLEBANOFF, P.S., 1955, Characteristics of turbulence in a boundary layer with zero pressure gradient, NACA Report No. 1247

KUAN,C.L. \& WANG, T.W., 1988, Some intermittent behavior of transitional boundary layers, submitted to Experimenial Thermal and Fluid Science.

MORKOVIN M.V 1978 , Instability, transition to turbulence and predictability, NATO AGARDograph No. 236

MUSKER, A.J., 1979, Explicit expression for the smooth wall velocity distribution in a turbulent boundary layer, $A I A A$ Journal, 17, No. 6, 655 .

SCHLICHTING' H. 1979 Boundary Layer Theory. 7th ed., McGraw Hill New York NY

SUDER, K.L., O'BRIEN, J'E, \& RESHOTKO, E, 1988 Experimental study of bypass transition in a boundary layer, NASA TM 100913 .

WANG, T., SIMON, T.W. \& BUDDHAVARAPU, J., 1985 Heat transfer and fiuid mechanics measurements in transitional boundary layer flows, ASME Journal of Engr. for Gas Turbines and Power. 107, 1007.

WYGNANSKI, I., SOKOLOV, M. \&'FRIEDMAN, D., 1976 On a turbulent spot in a laminar boundary layer,", J. Fluid Mech., 78, part 4,785. 


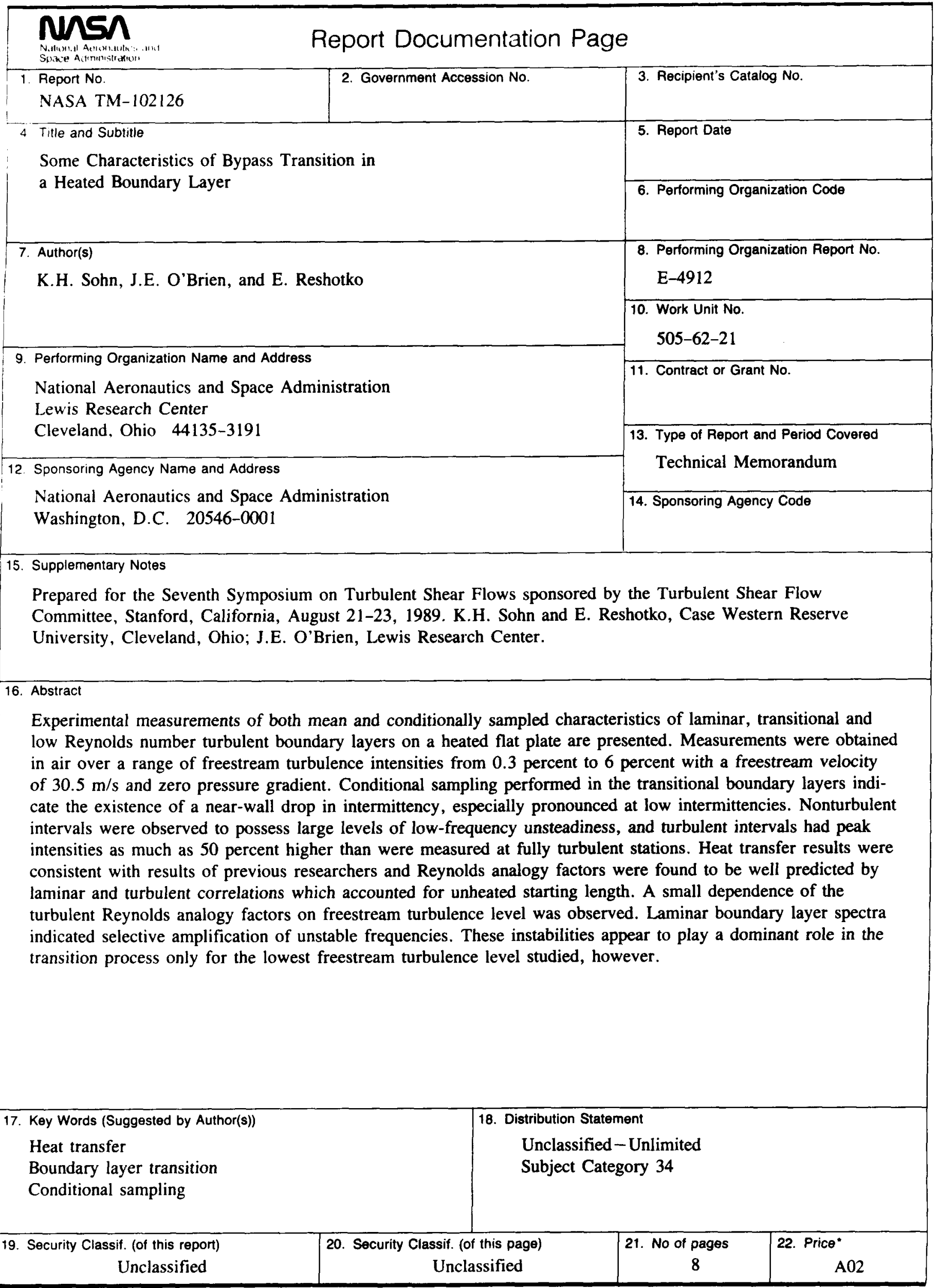

